\title{
Equivalent Volume Sources for Explosions at Depth: Theory and Observations
}

\author{
by Paul G. Richards and Won-Young Kim
}

\begin{abstract}
For simple theoretical models of an underground explosion, two apparently different relationships between isotropic moment and volume change have been published. We show that there is no inconsistency, because these relationships are based on two different definitions of volume change at the explosion source. We report observations of three 25-ton chemical explosions conducted in 1997 at the former nuclear test site near Semipalatinsk, Kazakhstan. These show the theoretically predicted decrease of signal strength as the shot depth increases. We argue that the relationship between isotropic moment and volume change proposed by Müller (1973) is preferred.
\end{abstract}

\section{Introduction}

For decades before the concepts of body force equivalents were developed, point sources of elastic waves were commonly characterized by nucleii of strain. For simple explosion point sources, the strain nucleus corresponds to the insertion of a finite volume of rock (of the same type as present at the shot point) into the medium effectively at the position of the point source. In modern seismology, with sources that add no linear or angular momentum, the theory for body force equivalents has been developed and widely applied in terms of moment tensors. Müller (1973) compared the two different approaches, and obtained the formula

$$
M_{\mathrm{I}}=(\lambda+2 \mu) \delta V,
$$

relating the isotropic moment $\left(M_{\mathrm{I}}\right)$ of an explosive point source to an equivalent volume change (here, $\delta V$ ), which he took to be the product of the surface area of a sphere of material surrounding the explosion, and the radius increase of this material as a result of the explosion. The constant of proportionality in equation (1) between $M_{\mathrm{I}}$ and $\delta V$ is $\lambda+$ $2 \mu=\rho \alpha^{2}$, in which $\rho$ is the mass density, $\alpha$ the $P$-wave speed, and $\lambda$ and $\mu$ the Lamé moduli. For wavelengths that are long compared to the diameter of the sphere surrounding the source and whose volume increases from $V$ to $V+\delta V$, $\delta V$ is an apparent volume change introduced effectively at a point in the medium, and generating only $P$-wave motions.

Müller's formula given in equation (1) is appealing because of its similarity to the result $M_{0}=\mu \delta V$ for the scalar moment $\left(M_{0}\right)$ of a double-couple point source (shear dislocation), where $\mu=\rho \beta^{2}, \beta$ is the $S$-wave speed, and $\delta V$ is the product of average fault area and average fault slip. Such a source radiates both $P$ - and $S$-waves, with the latter predominating.

We can interpret the basic formulas for $M_{\mathrm{I}}$ and $M_{0}$ in several different ways. For example, if the explosion is modeled as a pressure jump inside a spherical cavity whose radius is smaller than the shortest radiated wavelength of interest, then the radius increase (say, from $a$ to $a+\delta a$ ) is simply related to $\delta V$ by $\delta V=4 \pi a^{2} \delta a$, and the volume increase is the actual increase in cavity volume. This is the approach taken by many authors including Sharpe (1942), Latter et al. (1959, 1961), and Mueller and Murphy (1971). For the point source of faulting, $\delta V$ has been called the potency by Heaton and Heaton (1989) and Ben-Zion (2001), going back to ideas of Ben-Menahem et al. (1965), who chose a volume change rather than $M_{0}$ for characterizing the point-source strength of shearing sources (equivalent to their capability to excite long wavelength seismic waves).

Unfortunately, Müller's (1973) result, equation (1), seems to conflict with a relationship

$$
M_{\mathrm{I}}=[\lambda+(2 / 3) \mu] \Delta V
$$

between isotropic moment $M_{\mathrm{I}}$ and a volume change $\Delta V$ used by several authors to characterize the strength of explosion sources. For example, Doornbos (1977), Bowers and Hudson (1999), and Dreger et al. (2000), use equation (2) to relate isotropic moment and volume change, and some authors imply the merit of a relationship between this volume change and the yield of a well-tamped and fully contained explosion. Equation (2) is appealing, in part because the combination $\lambda+(2 / 3) \mu$ is just the bulk modulus.

The difference between equations (1) and (2) was first pointed out by Müller (2001), though he used the same symbol for volume change in these equations, and he regarded the two relationships between moment and volume change as inconsistent. He wrote that "the discrepancy ... is not 
resolved," and thus whether equation (1) or (2) is correct was left as an open question.

Our first goal in this paper is to show that there is no discrepancy. We do this by reviewing the derivations of equations (1) and (2), showing that the volume changes involved in these two equations are different. Both versions of the relationship between moment and volume change are correct.

Our second goal is to make a recommendation on which of the two volume changes is to be preferred for purposes of interpreting the seismic signal of underground explosions (chemical or nuclear). We do this in the context of interpreting the regional signals of three chemical explosions, each of 25 tons, conducted in 1997 at different depths in the same source region of the former nuclear test site near Semipalatinsk, Kazakhstan. The two different definitions of volume change ( $\delta V$ and $\Delta V$ ) associated with a particular isotropic moment are proportional to each other, so it might seem arbitrary as to which one to use. But, marginally, we prefer the definition of $\delta V$, used in equation (1) as given originally by Müller (1973), since it is a volume change that actually occurs, that has a clear kinematic definition, and that neatly matches the corresponding result for shear dislocations.

\section{Theory}

Many authors have developed a simple theory for the seismic waves emanating isotropically from an explosion in an infinite homogeneous isotropic elastic medium specified by its mass density $\rho$, and its Lamé moduli $\lambda$ and $\mu$. The main challenge is to relate near-source properties, such as a volume change or the strength of three mutually perpendicular dipoles, to the amplitude and pulse shape of far-field $P$ waves. A modern understanding is easily developed from first principles by use of the scalar $P$-wave potential $\phi$, in terms of which displacement $\mathbf{u}$ is given by $\mathbf{u}=\nabla \phi$, since no $S$-waves are generated. Here, $\phi$ is the outgoing spherically-symmetric solution to the scalar wave equation $\left(1 / \alpha^{2}\right)$ $\partial^{2} \phi / \partial t^{2}=\nabla^{2} \phi$. With $r$ as the radial coordinate in spherical polars, it follows that $\nabla^{2} \phi=\left(1 / r^{2}\right)(\partial / \partial r)\left[r^{2}(\partial \phi / \partial r)\right]=(1 /$ $r) \partial^{2}(r \phi) / \partial^{2} r$. From the well-known solution to the onedimensional wave equation $\left(1 / \alpha^{2}\right) \partial^{2}(r \phi) / \partial t^{2}=\partial^{2}(r \phi) / \partial^{2} r$, it follows that $\phi(r, t)=-F(t-r / \alpha) / r$ for some function $F$, called the reduced displacement potential. Then $(\partial / \partial r)$ $F=(-1 / \alpha)(d / d t) F$ and the radial displacement is given exactly by

$$
u_{r}=(d / d t)[F(t-r / \alpha)] /(\alpha r)+[F(t-r / \alpha)] / r^{2} .
$$

The first term dominates in the far field (when $r$ is large compared to the wavelength), as shown by Rayleigh (1877, article 279), and the second term dominates in the near field (when $r$ is less than a wavelength), for example at $r=a$, the radius of a small sphere centered on the source at $r=$ 0 . For static problems, in a sense there is no far field and all points are in the near field: only the second term is non-zero.
If $r=a$, then $u_{r}=\delta a$ for times sufficiently long that the final increase in radius has been attained. It follows from use of the last term in equation (3) that

$$
F(\infty)=a^{2} \delta a .
$$

In the case of a point-source explosion modeled by three mutually perpendicular dipoles the final (static) displacement appears first to have been obtained by Dougall (1898). His solution, quoted by Love $(1944,132)$ and many others, is

$$
u_{r}=\frac{1}{4 \pi(\lambda+2 \mu) r^{2}} M_{\mathrm{I}},
$$

using $M_{\mathrm{I}}$ for the strength of each dipole. Comparison between equation (5) and the last term of equation (3) relates $M_{\mathrm{I}}$ to $F$ as

$$
M_{\mathrm{I}}(t)=4 \pi(\lambda+2 \mu) F(t) .
$$

Though results equivalent to these have been known for several decades, Müller (1973) was the first to combine equations (4) and (6) as

$M_{\mathrm{I}}(\infty)=4 \pi(\lambda+2 \mu) a^{2} \delta a=(\lambda+2 \mu) \delta V, \quad(1$, again $)$

and to make the comparison with the usual shear dislocation formula relating earthquake scalar moment to a source volume given by slip times fault area. The result here depends directly on $\delta V$ and is independent of $a$ in the sense that it is only the product $a^{2} \delta a$, which influences source strength. (If, say, the value of $a$ were doubled, then for a given source the value of $\delta a$ would, according to equation (4), be four times smaller, in order that $F(\infty)=a^{2} \delta a$ would be unchanged. The independence of $\delta V$ on $a$ has been noted by many authors.)

In contrast to Müller's (1973) approach, Eshelby (1957) and others including Aki and Richards $(1980,2002)$ chose to separate the medium containing both the source and the seismic waves it generates into two regions: a source volume $V$, inside which there may be nonlinear behavior such as fracture and melting (for an explosion, rocks may even vaporize); and a region outside $V$ in which seismic waves propagate linearly. The theory has been developed for general volume sources. In application to the simple model of an explosion, Eshelby's approach (discussed next as a series of thought experiments that we describe in five steps) is like Müller's (1973), in that it entails a spherical source region which undergoes an expansion.

In the first step, the source region in Eshelby's approach is considered in its original state, and identification is made of a sphere of volume $V$, centered on the place which will become the explosion source point. The volume is sufficiently large to include all the material which will undergo 
strains larger than can be related to stress by Hooke's law. The spherical volume $V$ is removed from its surrounding matrix. Tractions are applied on both the internal surfacecall it $\Sigma^{+}$- of the matrix, and on the external surface $-\Sigma^{-}-$ of the sphere that has been removed, in order to maintain their shapes.

In the second step, the source region $V$ (inside $\Sigma^{-}$) undergoes a transformation, which does not change its stress. This is accomplished by a stress-free strain with cartesian components $\Delta e_{r s}$, which for our discussion of an explosion source is an isotropic expansion to a new volume $V+\Delta V$ where $\Delta V$ and the stress-free strain are related by $\Delta e_{r s}=$ $(1 / 3)(\Delta V / V) \delta_{r s}$.

In the third step, an additional stress field throughout the transformed volume is applied to return the surface $\Sigma^{-}$ to its original shape. In Eshelby's approach, strain and stress are still related by the same elastic moduli, so the additional stress field must be related to strain throughout the source volume by Hooke's law for an isotropic material: $-\Delta \tau_{p q}=$ $-\lambda \Delta e_{r r} \delta_{p q}-2 \mu \Delta e_{p q}$. It follows that to accomplish this step we merely need to apply a pressure given by $[\lambda+(2 /$ 3) $\mu](\Delta V / V)$ to the surface $\Sigma^{-}$(and hence throughout the source volume). Not surprisingly, the elastic modulus appearing here is $\lambda+(2 / 3) \mu$, equal to the bulk modulus.

In the fourth step we put the spherical source region back into its hole (noting that it has the correct shape to enable it to fit exactly), and weld it back in place so that there can be no sliding across its surface, and no development of any cavities. At this point there is still an extra traction on the surface $\Sigma^{-}$of the source region (the pressure described in step four) so that there is a traction difference between $\Sigma^{-}$and $\Sigma^{+}$.

In the fifth and final step, we release the applied traction on $\Sigma^{-}$. This is equivalent to imposing a traction discontinuity across the newly welded surface. As a result, the source region changes its size. We denote its new volume by $V+$ $\delta V$. Then, $\delta V$ is the actual volume change of Müller (1973). Aki and Richards (2002) analyze the displacement caused by the traction discontinuity and show that the resulting moment tensor $M_{p q}$ is given by the volume integral of $\Delta \tau_{p q}$ throughout the source volume, and hence in the present case is given by $M_{p q}=[\lambda+(2 / 3) \mu] \Delta V \delta_{p q}$. Since $\mathbf{M}$ is isotropic, we can express this result in terms of the scalar explosion moment as

$$
M_{\mathrm{I}}=[\lambda+(2 / 3) \mu] \Delta V . \quad \text { (2, again) }
$$

There is no discrepancy between equations (1) and (2) once it is realized that the two volume changes, denoted here as $\delta V$ and $\Delta V$, have different definitions. They are proportional to each other, with $\Delta V / \delta V=(\lambda+2 \mu) /[\lambda+(2 / 3) \mu]=1.8$ if $\lambda=\mu$. In general, $\Delta V$ is significantly larger than $\delta V$ because it is an unconfined volume change.

Combining equations (1) or (2) with the far-field term in equation (3), we see that the far-field $P$-waves are proportional to the rate of change of volume increase in the source region. This conclusion is very helpful in developing an intuitive understanding of how conditions at the source can influence the strength of the radiated $P$-waves. In particular, the depth-of-burial of an explosive source influences the signal strength, as discussed for example by Mueller and Murphy (1971). The deeper the source, the greater the confining pressure due to overburden. For a series of explosions of fixed yield, fired at different depths but otherwise in conditions that as nearly as possible are the same from shot to shot, we therefore expect the deeper shots to have smaller seismic signals, because their volume increase $\delta V$ will be smaller due to the greater confining pressure. (We are assuming a series in which all shots are tamped and fired at depths great enough to achieve containment of the crushed and perhaps vaporized products of the explosion. The fact that the far-field $P$ displacement amplitude is proportional to rate of change of volume increase, implies that this displacement is itself a pulse whose area-under the pulse-is proportional to the volume increase.)

In the next section we describe data taken from a series of shots, which approximately meet the conditions necessary to study depth-of-burial effects experimentally. A final section discusses whether the $\delta V$ or $\Delta V$ definition of volume change is more helpful to our understanding of these results.

\section{Observations}

During August-September, 1997, three 25-ton chemical explosions were conducted at different depths of burial- 50 , 300 , and $550 \mathrm{~m}$-at the former Soviet Union's Semipalatinsk Nuclear Test Site in Eastern Kazakhstan (Leith and Kluchko, 1998). The location, origin time, and total charge weight of the three explosions are listed in Table 1. A map of shot locations and stations at regional distances is given in Figure 1.

This project had as a goal the destruction of shafts originally constructed in the Soviet era for nuclear weapons testing. The process of destruction was turned into a depth-ofburial experiment, in which studies of the resulting seismic signals were done to investigate the effects of different depths of shots having the same charge.

The boreholes for the three different shots were cylindrical-about one meter in diameter-and the 25-ton chemical explosive occupied about $34 \mathrm{~m}$ of shaft length. The explosive was poured into confined sections of the boreholes at the designed depth, and detonated all at once. The blasts were initiated by a detonator located at the center of the 34meter-long explosive column. The explosive was a granulated waterproof form of tri-nitro toluene (TNT) called "Granulotol" having a detonation speed of about 5.5 to 6.5 $\mathrm{km} / \mathrm{sec}$. The sources were fully tamped by filling the borehole with a mix of sand and gravel.

Denny (1998) analyzed local data recorded at distance ranges of 4 to $17 \mathrm{~km}$ from the depth-of-burial experiment, and concluded that Sharpe's (1942) simple spherically symmetric model is an adequate representation of the seismic- 
Table 1

Source Information for Three Chemical Explosions at Different Depths of Burial

\begin{tabular}{lccccccc}
\hline Date & $\begin{array}{c}\text { Origin Time } \\
(\text { hh:mm:sec })\end{array}$ & $\begin{array}{c}\text { Lat. } \\
\left({ }^{\circ} \mathrm{N}\right)\end{array}$ & $\begin{array}{c}\text { Long. } \\
\left({ }^{\circ} \mathrm{E}\right)\end{array}$ & $\begin{array}{c}\text { Depth } \\
(\mathrm{m})\end{array}$ & $\begin{array}{c}\text { Magnitude } \\
\left(M_{\mathrm{L}}\right)\end{array}$ & $\begin{array}{c}\text { Charge } \\
(\text { ton })\end{array}$ & $\begin{array}{c}\text { Shot } \\
\text { Hole }\end{array}$ \\
\hline 1997 Aug 03 & $08: 04: 20.04$ & 49.9781 & 78.8200 & 50 & 2.35 & 25 & 1311 \\
1997 Aug 31 & $07: 08: 39.26$ & 49.8837 & 78.8148 & 300 & 2.09 & 25 & 1381 \\
1997 Sept 28 & $07: 30: 15.13$ & 49.8802 & 78.7587 & 550 & 1.95 & 25 & 1349 \\
\hline
\end{tabular}

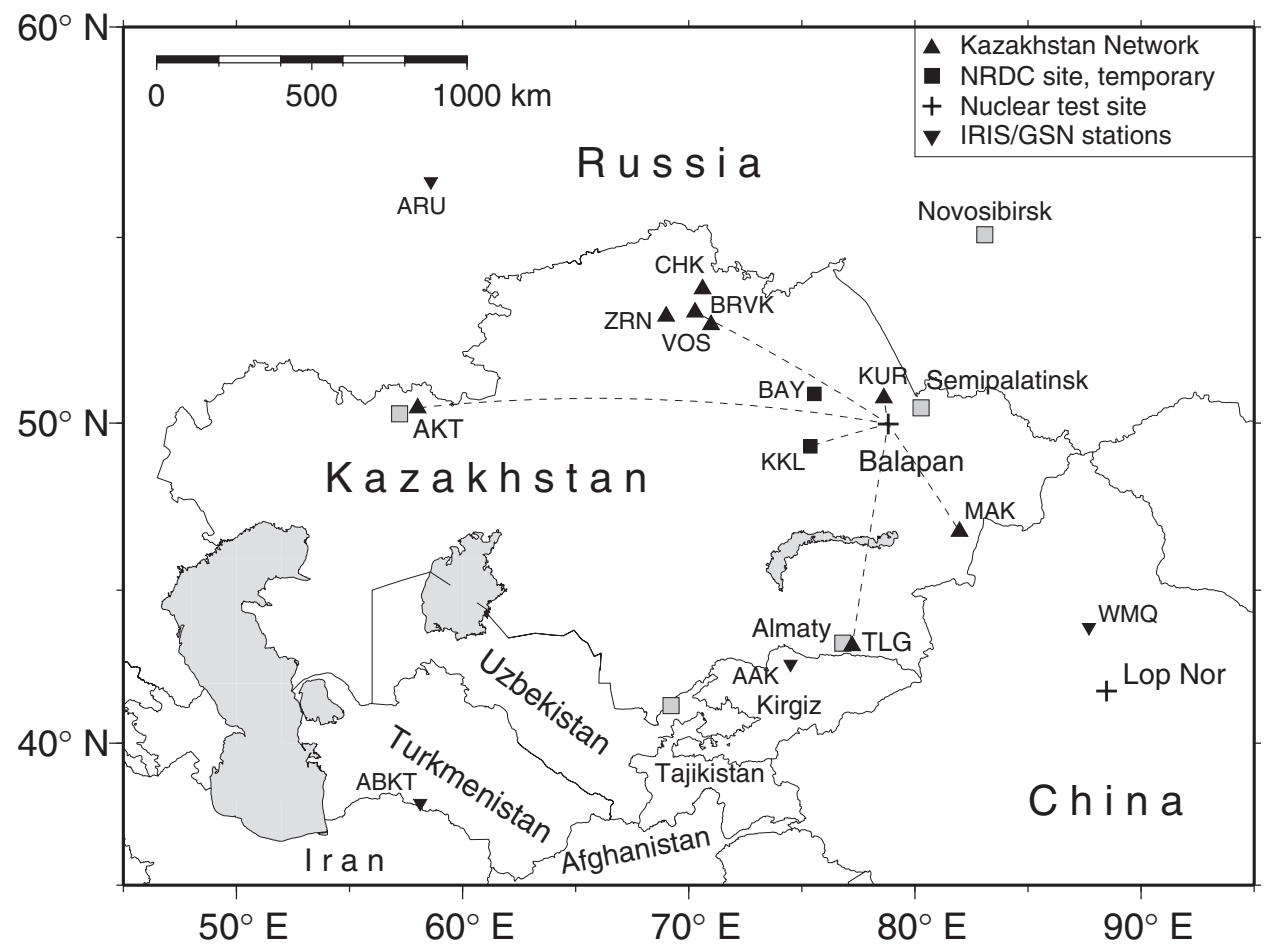

Figure 1. Locations of eight broadband seismographic stations of the Kazakhstan Network, closed triangles. Global Seismic Network (GSN) stations, inverted triangles; underground nuclear test sites, crosses. Two stations installed in 1987 by the Natural Resources Defense Council (NRDC) in 1987 at temporary sites (BAY, KKL) were reinstalled for the depth-of-burial experiment. Wave propagation paths between shot points and seismographic stations are indicated by dotted lines.

source function for these underground explosions. A more realistic cylindrical cavity model for chemical explosions such as these 25-ton depth-of-burial shots was given by AboZena (1977). Glenn et al. (1986) showed that the source corner frequency for underground explosions increases with depth-of-burial, whereas the seismic moment decreases.

Figure 2 shows vertical-component seismic records at the nearest station KUR (Kurchatov; distance $=83-94 \mathrm{~km}$, azimuth $=350-354^{\circ}$ ) from the three explosions. These records clearly show that the seismic signals from the explosion set off at shallowest depth of $50 \mathrm{~m}$ are strongest, and the signals from the explosion set off at the greatest depth $(550 \mathrm{~m})$ are weakest among the three sets of records. As discussed in the previous section, this change in signal strength is due to the increase of overburden with depth, leading to smaller actual volume change, $\delta V$, of the nonlinear source region for the deeper explosions. Seismic records from about 10 broadband seismographic stations at distance ranges of 83-760 km in Kazakhstan all show seismic amplitude decreasing with explosion depth, as noted by Myers et al. (1999).

Peak amplitudes, measured on simulated WoodAnderson vertical-component records from the three explosions, are shown in Figure 3. Using an amplitude attenuation curve for the northeastern U.S. (Kim, 1998), the local magnitudes of these shots are estimated to be 2.5, 2.2, and 2.0, for 50,300 , and $550 \mathrm{~m}$ depth, respectively. If we limit the peak amplitude measurements in the $P$-wave window, then the estimated local magnitudes are $2.35,2.09$, and 1.95 , for 50,300 , and $550 \mathrm{~m}$ depth, respectively (Table 1).

There is a practical complication in the interpretation of these magnitudes in terms of the theory reviewed in the pre- 

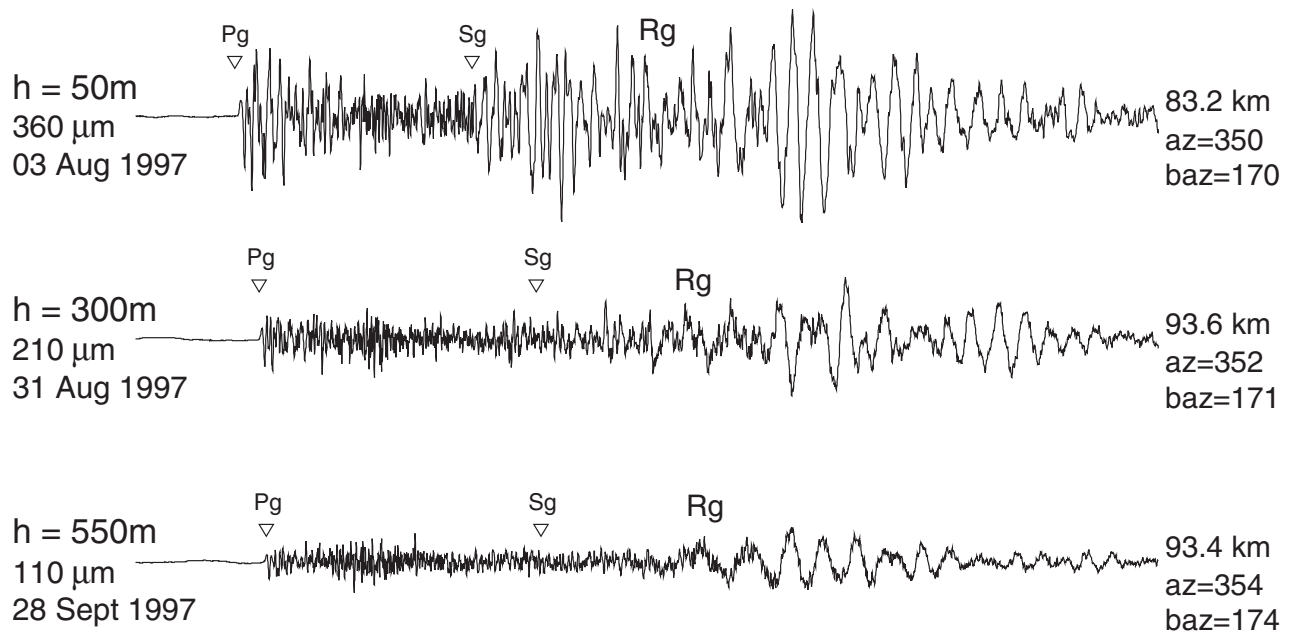

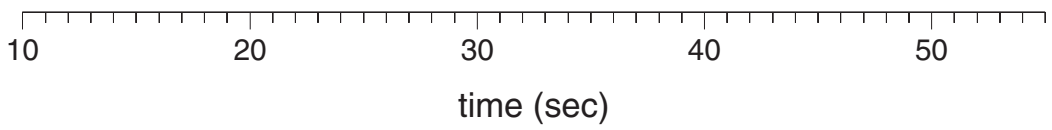

Figure 2. Vertical-component Wood-Anderson records at KUR (distance $=83-93$ $\mathrm{km}$, azimuth $=350-354^{\circ}$ ) from three 25 -ton explosions at 50,300, and $550 \mathrm{~m}$ depths at Balapan, on the former Soviet nuclear test site near Semipalatinsk, Kazakhstan. Note that the phase denoted as $S g$ appears to be a $P$-to- $S$ phase converted at the free surface. True direct $S$ waves are not excited by the explosive source in an isotropic medium, and hence $S g$ amplitudes decay as $P g$ for various source depths.

vious section, because the data include near-source interactions with the free surface, and effects during propagation due to the Moho, and perhaps due to other interfaces and layers. (The free surface typically amplifies signals at the recording station [Kim et al. 1997], but this effect is the same for all explosions, and free surface corrections at the receiver are not an issue in this article.) In order to minimize nearsource effects of the free surface on seismic wave amplitudes, which will be more significant for signals from shallower burial depths, we measured zero-to-peak amplitudes (one-half the peak-to-peak) of the first arrival $P$-waves at three stations: KUR; VOS (Vostochnaya; distance $=626 \mathrm{~km}$, azimuth $=302^{\circ}$ ); and ZRN (Zerenda; distance $=758 \mathrm{~km}$, azimuth $=300^{\circ}$ ) as shown in Figure 4 . The first arrival $P$ wave amplitudes are plotted in Figure 3 for these three stations, for explosions at the three different depths. This figure shows very clearly the increasing signal strength as the depth of the shot decreases.

\section{Discussion and Conclusions}

It is gratifying to see such agreement between theory and observations, in the way that signal amplitudes (Fig. 3) decrease with increasing depth-of-burial. Such observations support the idea that the seismic source strength of an explosion can usefully be characterized by the volume increase at the source. But, which of the definitions, $\delta V$ or $\Delta V$, is preferred?

The definition of $\Delta V$, given in the second step of the five steps described earlier, may seem appealing in that it is associated with a stress-free strain that phenomenologically appears to describe the consequences of the explosion in an unconfined volume. It is on this basis that it might seem appropriate to establish a connection between $\Delta V$ and the explosion yield. But this appeal is weakened by the fact that $\Delta V$, in the practical interpretation of explosions with the same charge size at different depths (such as we have described in the previous section), is different for the different explosions. As we noted, above, $\delta V$ and $\Delta V$ are proportional to each other, so they must both be depth dependent. Although the source region $V$ (inside $\Sigma^{-}$) has, in the first step of Eshelby's approach, conceptually been removed from the medium, the stress-free strain it undergoes in the second step leads to a volume change $\Delta V$ that must be influenced by the confining pressure into which the source volume will be returned and released in steps four and five. The volume change associated with stress-free strain is therefore, in practice, not a suitable candidate for a relationship to yield that is independent of source depth.

In contrast, $\delta V$ is a volume change that actually occurs and has a clear kinematic definition (the expansion $4 \pi a^{2}$ $\delta a$ where $\delta a$ is proportional to $1 / a^{2}$ ) that we can more easily appreciate will depend on source depth. This definition directly indicates, for a series of well-tamped and fully contained explosions of the same charge size but fired at different depths, that deeper explosions will have weaker seismic signals (because $\delta a$ at fixed $a$ will be smaller for greater confining pressure). In contrast the volume change symbol- 


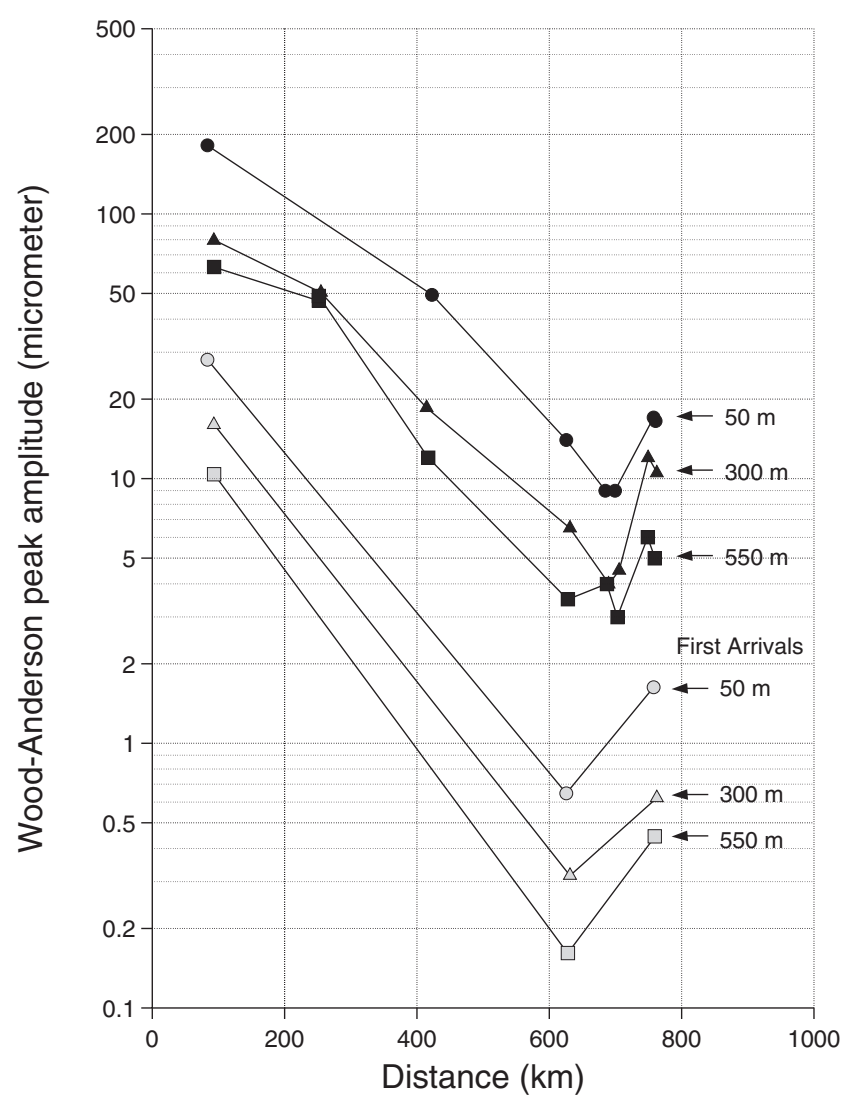

Figure 3. Peak amplitude of the ground motions from three explosions set off at different burial depths are plotted against distance. Amplitudes are measured from simulated Wood-Anderson vertical records and are plotted with filled circles (50 $\mathrm{m}$ depth), filled triangles (300 $\mathrm{m}$ depth), and filled squares $(550 \mathrm{~m}$ depth). Peak amplitude of the first arrival $P$ waves in micrometers measured from simulated Wood-Anderson vertical records at three stations are plotted with shaded symbols; the amplitudes are plotted with one quarter of their values to avoid crowding.

ized by $\Delta V$ is hypothetical, and requires several steps before it can be used to predict the weakening of seismic signals for explosions of greater depth. We therefore conclude that the volume change $\delta V$ proposed by Müller (1973) is preferable for characterizing seismic source strength of underground explosions. Another attraction of this definition of volume change is that Müller's relationship $M_{\mathrm{I}}=(\lambda+2 \mu)$ $\delta V$ closely parallels the familiar relationship between seismic moment and potency of an earthquake modeled by a shear dislocation. Finally, we note for explosion sources that the relationship between volume change and yield will depend on source depth.

\section{Acknowledgments}

This article is adapted from a presentation given in January 2003 at a colloquium in Germany in memory of Gerhard Müller. P.G.R. appreciates the all-too-few opportunities he had from 1971 to 2001 to discuss source theory and wave propagation with Dr. Müller. Discussion related to this

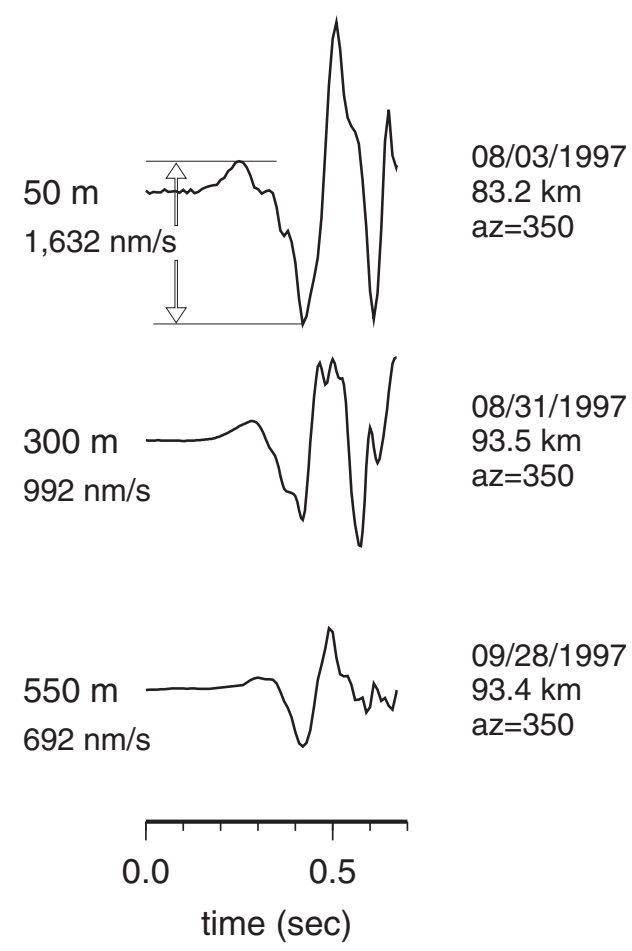

Figure 4. The first arrival $P$ waves on verticalcomponent broadband records from the 25-ton explosions at 50, 300, and $550 \mathrm{~m}$ depths, as recorded at KUR, are plotted. Depth-of-burial and peak-to-peak amplitude in $\mathrm{Nm} / \mathrm{sec}$ are indicated at the beginning of each trace. Arrows on the top trace indicate the range of the peak-to-peak measurement for the first part of the arriving signal.

article appears in Problem 3.8 of Aki and Richards (2002). Our Kazakhstan data were obtained with support from the Lawrence Livermore National Laboratory, subcontract B336507, and Department of Defense contract DSWA01-97-C0156. Permanent broadband seismographic stations in Kazakhstan used to acquire data from the depth-of-burial experiment were supported by the International Science and Technology Center, Project K63, and the IRIS Consortium. Publication is supported in part by the Air Force Research Laboratory contract F19628-03-C-0129. This is LamontDoherty Earth Observatory contribution number 6738.

\section{References}

Abo-Zena, A. M. (1977). Radiation from a finite cylindrical explosive source, Geophys. 42, 1384-1393.

Aki, K., and P. G. Richards (1980). Quantitative Seismology: Theory and Methods, First Ed., W. H. Freeman, San Francisco.

Aki, K., and P. G. Richards (2002). Quantitative Seismology, Second Ed., University Science Books, Sausalito, California.

Ben-Menahem, A., S. W. Smith, and T.-L. Teng (1965). A procedure for source studies from spectrums of long-period seismic body waves, Bull. Seism. Soc. Am. 55, 203-235.

Ben-Zion, Y. (2001). On quantification of the earthquake source, Seism. Res. Lett. 72, 151-152.

Bowers, D., and J. A. Hudson (1999). Defining the scalar moment of a seismic source with a general moment tensor, Bull. Seism. Soc. Am. 89, 1390-1394.

Denny, M. D. (1998). Seismic-source corner frequencies from the depth of burial experiment, Lawrence Livermore National Laboratory, Livermore, California, UCRL-ID-132296, November 1998, 6 pp. 
Doornbos, D. J. (1977). The excitation of normal modes of the Earth by sources with volume change, Geophys. J. R. Astr. Soc. 51, 465-474.

Dougall, J. (1898). A general method of solving the equations of elasticity, Edinburgh Math. Soc. Proc. 16, 82-98.

Dreger, D. S., H. Tkalčić, and M. Johnston (2000). Dilatational processes accompanying earthquakes in the Long Valley Caldera, Science 288, $122-125$.

Eshelby, J. D. (1957). The determination of the elastic field of an ellipsoidal inclusion, and related problems, Proc. R. Soc. Lond. A241, 376-396.

Glenn, L. A., B. Moran, A. J. C. Ladd, K. A. Wilson, and J. A. Rial (1986). Elastic radiation from an explosively-loaded axisymmetric cavity, Geophys. J. R. Astr. Soc. 86, 119-136.

Heaton, T. H., and R. E. Heaton (1989). Static deformation from point forces and force couples located in welded elastic Poissonian halfspaces: implications for seismic moment tensors, Bull. Seism. Soc. Am. 79, 813-841.

Kennett, B. L. N., and R. Engdahl (1991). Traveltimes for global earthquake location and phase identification, Geophys. J. Int. 105, 429-465.

Kim, W.-Y. (1998). The $M_{L}$ scale in Eastern North America, Bull. Seism. Soc Am. 88, 935-951.

Kim, W.-Y., V. Aharonian, A. L. Lerner-Lam, and P. G. Richards (1997). Discrimination of earthquakes and explosions in southern Russia using regional high-frequency three-component data from the IRIS/JSP Caucasus Network, Bull. Seism. Soc. Am. 87, 569-588.

Kim, W.-Y., V. V. Kazakov, A. G. Vanchugov, and D. W. Simpson (1996). Broadband and array observations at low noise sites in Kazakhstan: opportunity for seismic monitoring of the Comprehensive Test Ban Treaty, in Monitoring a Comprehensive Test Ban Treaty, E. Husebye and A. M. Dainty (Editors), Kluwer Academic Publishers, Norwell, Massachusetts, 462-482.

Latter, A. L., R. E. LeLevier, E. A. Martinelli, and W. G. McMillan (1961). A method of concealing underground nuclear explosions, J. Geophys. Res. 66, 943-946.
Latter, A. L., E. A. Martinelli, and E. Teller (1959). A seismic scaling law for underground explosions, Phys. Fluids 2, 280-282.

Leith, W., and L. J. Kluchko (1998), Seismic experiments, nuclear dismantlement go hand in hand in Kazakstan, EOS, Transactions, Amer. Geophys. Union 79, 437, 443-444.

Love, A. E. H. (1944). A Treatise on the Mathematical Theory of Elasticity, Dover Publications, New York.

Mueller, R. H., and J. R. Murphy (1971). Seismic characteristics of underground nuclear detonations: part I, seismic spectrum scaling, Bull. Seism. Soc. Am. 61, 1675-1692.

Müller, G. (1973). Seismic moment and long-period radiation of underground nuclear explosions, Bull. Seism. Soc. Am. 63, 847-857.

Müller, G. (2001). Volume change of seismic sources from moment tensors, Bull. Seism. Soc. Am. 91, 880-884.

Myers, S., W. R. Walter, K. Mayeda, and L. Glenn (1999). Observations in support of $R g$ scattering as a source for explosion $S$ waves: regional and local recordings of the 1997 Kazakhstan depth of burial experiment, Bull. Seism. Soc. Am. 89, 544-549.

Rayleigh, J. W. S. (1877). The Theory of Sound, First Ed.; also in the second (1894) edition, republished by Dover (1945).

Sharpe, J. A. (1942). The production of elastic waves by explosion pressures: part I, theory and empirical field observations, Geophys. 7, $144-154$.

Lamont-Doherty Earth Observatory of Columbia University

Palisades, New York 10964

(P.G.R., W.-Y.K.)

Department of Earth and Environmental Sciences, Columbia University New York, New York

(P.G.R.)

Manuscript received 26 February 2004. 